\title{
Supraventricular tachycardia after nebulized salbutamol therapy in a neonate: Case report
}

\author{
Birgul Say $M D^{a}$, Halil Degirmencioglu $M D^{a}$, Hayriye Gozde Kanmaz Kutman $M D^{a}$, Nurdan Uras $M D^{a}$ and \\ Ugur Dilmen $M D^{a, b}$
}

\begin{abstract}
Supraventricular tachycardia (SVT) is the most common sustained arrhythmia in neonates and infants. Presentation of SVT in the neonate is usually subtle and frequently complicated by congestive heart failure. Despite the widespread use of $\beta 2$-agonists, their safety has been questioned. Several studies have reported an increased incidence of cardiac arrhythmias in patients treated with these agents, and other studies have found increased rates of cardiovascular death associated with the use of oral and nebulized $\beta 2$-agonists such as salbutamol, which is used to treat bronchospasm in newborns with several diseases. Herein, we report a case of SVT following administration of nebulized salbutamol in a neonate.

Keywords: tachycardia supraventricular, salbutamol, newborn.
\end{abstract}

http:/ /dx.doi.org/10.5546/aap.2015.eng.e98

\section{INTRODUCTION}

Supraventricular tachycardia (SVT) includes all forms of tachycardia originated above the bifurcation of the bundle of His or have mechanisms dependent on the bundle of His. SVT is the most common sustained arrhythmia in neonates and infants. Predisposing factors such as congenital heart disease, drug administration, illness, and fever occur only in $15 \%$ of cases. ${ }^{1}$ The presentation of SVT in the neonate is frequently subtle and it may include pallor, cyanosis, restlessness, irritability, feeding difficulty, tachypnea, diaphoresis and grunting. SVT in newborns is frequently complicated by congestive heart failure, characterized by a fixed heart rate usually greater than 230 beats per minute. Typical electrocardiogram (ECG) findings of SVT include narrow QRS complexes, regular R-R

a. Division of Neonatology, Zekai Tahir Burak Maternity Teaching Hospital.

b. Department of Pediatrics, Yıldırım Beyazıt University School of Medicine

Ankara,Turkey.

E-mail Address:

Halil Degirmencioglu MD: hdegirmencioglu@gmail.com

Conflict of Interest: None.

Received: 7-14-2014

Accepted: 11-3-2014 intervals, and absent $\mathrm{P}$ waves or an abnormal $\mathrm{P}$ wave axis. Salbutamol, which has been used as bronchodilators for the prevention and treatment of chronic lung disease in preterm infants, for emergency treatment of hyperkalemia, and transient tachypnea of the newborn (TTN), ${ }^{2-4}$ is a direct-acting sympathomimetic agent that has a selective $\beta$-adrenergic effect. Because $\beta$-receptors in the heart are mainly the $\beta-1$ type, it is believed that salbutamol has minimal cardiovascular effects; however, tachycardia and cardiac arrhythmias have been reported as complications of salbutamol administration. ${ }^{5}$ Although well documented in adults, cardiac arrhythmias have not been widely reported in pediatric patients as a complication of salbutamol therapy.

This paper presents the case of an infant who was admitted to the neonatal intensive care unit (NICU) with a diagnosis of meconium aspiration syndrome and who developed multiple SVTs following administration of salbutamol during the recovery phase of her underlying illness.

\section{CASE REPORT}

The patient was a girl born to a 28-year-old mother via a spontaneous vaginal delivery after a term gestation; the birth weight was $3600 \mathrm{~g}$. The mother was healthy and had an uneventful pregnancy. The baby was delivered through thick meconium and she was cyanotic. Her mouth was suctioned and she was intubated and suctioned for thick, particulate meconium. Her pulse fell below 100 beats per minute (bpm) and positivepressure ventilation (PPV) was initiated. The patient's first and fifth Apgar scores were 5 and 6, respectively and she was transferred to the NICU. On admission to the NICU, the infant had a pulse of $157(\mathrm{bpm})$, a respiration rate of 72 per minute, and a mean arterial blood pressure of $34 \mathrm{mmHg}$. She remained with $100 \%$ oxygen. A capillary blood gas test showed a $\mathrm{pH}$ of 7.31, a $\mathrm{PCO}_{2}$ of $43 \mathrm{mmHg}$, and a $\mathrm{SpO}_{2}$ of $94 \%$ at 20 minutes of life, and she was severely tachypneic. After intubation, a blood culture was taken, a sepsis evaluation was performed, and intravenous (IV) antibiotics (crystalline penicilline; 50,000 
units / kg/day in divided doses every 12 hours, and gentamicine $4 \mathrm{mg} / \mathrm{kg}$ /day every 24 hours) were begun. Chest radiography revealed dense bilateral infiltrates with prominent air bronchograms. An echocardiogram at 10 hours of life revealed septal hypertrophy with flattening of the ventricular septum, consistent with persistent pulmonary hypertension of the neonate (PPHN). Treatment was aimed at interrupting the cycle of pulmonary vasoconstriction and hypoxia. Her chest radiographs started to demonstrate a reticulogranular pattern, and there was concern that she might have an element of surfactant deficiency.

Over the next several days, the need to ventilate and oxygenate the patient gradually decreased, and on day 7 of life, she was extubated to a nasal cannula. She had coarse bilateral breath sounds with marked expiratory wheezing. The cardiovascular examination was unremarkable, the echocardiographic examination was normal, and serum potassium and the other electrolytes were in the normal ranges at this time. Inhaled salbutamol therapy was initiated at a dose of $0.15 \mathrm{mg} / \mathrm{kg}(0.45 \mathrm{mg})$ every 4 hours. Following the inhalation of salbutamol solution (Ventolin nebules ${ }^{\circledR} 50$, Glaxo Wellcome; Greenford, UK), the infant's heart rate increased suddenly to $314 \mathrm{bpm}$. The ECG showed a narrow complex tachycardia at a heart rate of $310 \mathrm{bpm}$ and retrograde conducted $\mathrm{P}$ waves buried in the ST segment at an RP interval (Figure 1). Adenosine was administered via IV rapid bolus at a dose of $100 \mathrm{mcg} / \mathrm{kg}$ which was repeated twice. However, we were not able to obtain sinus rhythm despite the raised dose for the two subsequent

FIGURE 1. Electrocardiogram indicative of supraventricular tachycardia, with no visible $P$ wave and normal $Q R S$ morphology.

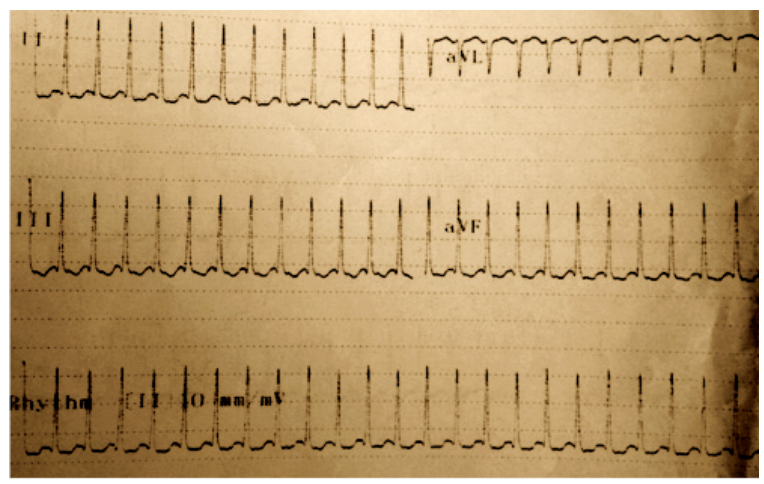

administrations of adenosine treatment and electrical cardioversion was performed, which terminated the SVT. Salbutamol treatment was ceased and SVT was never observed again. The patient was doing well and was discharged from the hospital on day 15 of life.

\section{DISCUSSION}

Despite the widespread use of $\beta 2$-agonists, their safety has been questioned. Existing data on the effects of these agents, especially those administered in an inhaled form, on myocardial electrophysiological properties are rare ${ }^{6}$ Pharmacologically, however, inhaled salbutamol can result in significant changes in cardiac electrophysiological properties. ${ }^{6}$ Salbutamol has been found to enhance atrioventricular nodal conduction and to decrease atrioventricular nodal, atrial, and ventricular refractoriness in addition to its positive chronotropic effects. $\beta 2$-agonists also increase QT dispersion. All of these alterations theoretically could contribute to the generation of tachycardia and tachyarrhythmias. Several studies have reported an increased incidence of cardiac arrhythmias in patients treated with the use of oral and nebulized $\beta 2$-agonists. ${ }^{5-7}$ $A$ recent human study demonstrated that salbutamol, a selective $\beta 2$-agonist, administered by nebulizer has significant electrophysiological effects on the atrium, nodes, and ventricle. Some studies found that salbutamol produced more evident changes on the electrophysiologic properties of the sinus node compared with the atrioventricular node. ${ }^{6,8}$ The cardiac effects of $\beta 2$-agonists include tachycardia, atrial and ventricular ectopic complexes, and atrial and ventricular arrhythmias. ${ }^{6,9}$

Although adverse drug reactions including tremor $(90 \%)$, hypokalaemia $(45.5 \%)$, and supraventricular tachycardia $(21 \%)$ were reported to be common, especially after administration of continuous intravenous infusion of salbutamol, ${ }^{10}$ the estimated SVT rate in children treated with inhaled $\beta 2$-agonists remains unknown. Tachycardia and tachyarrhythmias are very common in critically ill adult patients with various conditions. ${ }^{11}$ Predisposing factors for SVT include congenital heart disease, fever, and sympathomimetics. ${ }^{8,12}$ Our patient had no history of congenital heart disease or fever. Although she required brief PPV in the delivery room and respiratory support and she suffered from mild PPHN, her myocardial functions and cardiac output were always within normal limits, and 
no signs were observed of myocardial ischemia, which could cause a tendency toward arrhythmia. Acute management of SVT in children involves the use of vagal maneuvers or IV adenosine. IV adenosine has been found to be safe and highly effective in the management of SVT in infants and children. ${ }^{5}$

Two previous patient reports have described salbutamol-induced SVT in children. In the first report, SVT in a 19-month-old baby was converted by applying facial ice, and in the second case, a 4-year-old patient recovered with IV adenosine. ${ }^{5,6} \mathrm{We}$ initially administered a $100 \mathrm{mcg} / \mathrm{kg}$ dose of IV adenosine, and despite two repeated doses of $200 \mathrm{mcg} / \mathrm{kg}$, we could not obtain sinus rhythm. Cardioversion was performed with $2 \mathrm{j} / \mathrm{kg}$ followed by $4 \mathrm{j} / \mathrm{kg}$, and this treatment was successful.

Although nebulized, short-acting $\beta 2$-agonists are effective bronchodilator drugs that are widely prescribed for the treatment of airflow obstruction and increasingly for TTN and hyperkalemia in NICUs, there is considerable concern about their potential side effects, particularly those pertaining to heart rate and arrhythmias. In addition, $\beta 2$ agonists are widely used to treat neonates with bronchopulmonary dysplasia, but there is no evidence of their efficacy.

Therefore, we suggest that all infants should be carefully monitored for tachycardia which requires urgent treatment after nebulized salbutamol in both ambulatory and inpatient care, even if there are no underlying causes. Underlying causes that might facilitate arrhythmia should be evaluated.

\section{REFERENCES}

1. Moak JP. Supraventricular tachycardia in the neonate and infant. Prog Pediatr Cardiol 2000;11(81):25-38.

2. ArmangilD, YurdakökM, KorkmazA, YigitS, etal. Inhaled beta-2 agonist salbutamol for the treatment of transient tachypnea of the newborn. J Pediatr 2011;159(3):398-403.

3. Ng G, da Silva O, Ohlsson A. Bronchodilators for the prevention and treatment of chroniclung disease in preterm infants. Cochrane Database Syst Rev 2012;6:CD003214.

4. Vemgal P, Ohlsson A. Interventions for non-oliguric hyperkalaemia in preterm neonates. Cochrane Database Syst Rev 2012;5:CD005257.

5. DuaneM,ChandranL,MorelliPJ.Recurrentsupraventricular tachycardia as a complication of nebulized albuterol treatment. Clin Pediatr (Phila) 2000;39(11):673-7.

6. Kallergis EM, Manios EG, Kanoupakis EM, Schiza SE, et al. Acute electrophysiologic effects of inhaled salbutamol in humans. Chest 2005;127(6):2057-63.

7. Salpeter SR, Ormiston TM, Salpeter EE. Cardiovascular effects of $\beta$-agonists in patients with asthma and COPD: a meta-analysis. Chest 2004;125(6):2309-21.

8. Lowe MD, Rowland E, Brown MJ, Grace AA. Beta(2) adrenergicreceptorsmediateimportantelectrophysiological effects in human ventricular myocardium. Heart 2001;86(1):45-51.

9. Keller KA, Bhisitkul DM. Supraventricular tachycardia: a complication of nebulized albuterol. Pediatr Emerg Care 1995;11(2):98-9.

10. Habashy D, Lam LT, Browne GJ. The administration of beta2-agonists for paediatric asthma and its adverse reaction in Australian and New Zealand emergency departments: a cross-sectional survey. Eur J Emerg Med 2003;10(3):219-24.

11. Khorfan FM,SmithP, WattS, Barber KR. Effects of nebulized bronchodilator therapy on heart rate and arrhythmias in critically ill adult patients. Chest 2011;140(6):1466-72.

12. FlattA, CraneJ,PurdieG, Kwong T, etal. The cardiovascular effects of beta adrenergic agonist drugs administered by nebulisation. Postgrad Med J 1990;66(772):98-101. 